\title{
Design and Simulation Platform for Evaluation of Grid Distribution System and Transactive Energy
}

\author{
Himanshu Neema \\ himanshu.neema@vanderbilt.edu \\ Vanderbilt University \\ Nashville, TN, USA \\ Carlos Barreto \\ carlos.barreto@vanderbilt.edu \\ Vanderbilt University \\ Nashville, TN, USA
}

\author{
Harsh Vardhan \\ harsh.vardhan@vanderbilt.edu \\ Vanderbilt University \\ Nashville, TN, USA \\ Xenofon Koutsoukos \\ xenofon.koutsoukos@vanderbilt.edu \\ Vanderbilt University \\ Nashville, TN, USA
}

\begin{abstract}
With the advent of remarkable development of solar power panel and inverter technology and focus on reducing greenhouse emissions, there is increased migration from fossil fuels to carbon-free energy sources (e.g., solar, wind, and geothermal). A new paradigm called Transactive Energy (TE) [3] has emerged that utilizes economic and control techniques to effectively manage Distributed Energy Resources (DERs). Another goal of TE is to improve grid reliability and efficiency. However, to evaluate various TE approaches, a comprehensive simulation tool is needed that is easy to use and capable of simulating the power-grid along with various grid operational scenarios that occur in the transactive energy paradigm. In this research, we present a web-based design and simulation platform (called a design studio) targeted toward evaluation of powergrid distribution system and transactive energy approaches [1]. The design studio allows to edit and visualize existing power-grid models graphically, create new power-grid network models, simulate those networks, and inject various scenario-specific perturbations to evaluate specific configurations of transactive energy simulations. The design studio provides (i) a novel Domain-Specific Modeling Language (DSML) using the Web-based Generic Modeling Environment (WebGME [4]) for the graphical modeling of powergrid, cyber-physical attacks, and TE scenarios, and (ii) a reusable cloud-hosted simulation backend using the Gridlab-D power-grid distribution system simulation tool [2].
\end{abstract}

\section{CCS CONCEPTS}

- Computing methodologies $\rightarrow$ Modeling and simulation; - Computer systems organization $\rightarrow$ Embedded and cyberphysical systems.

\section{KEYWORDS}

Smart-grids, power-grid simulation, transactive energy, modeling and simulation, power-grid security and resilience

Permission to make digital or hard copies of part or all of this work for personal or classroom use is granted without fee provided that copies are not made or distributed for profit or commercial advantage and that copies bear this notice and the full citation on the first page. Copyrights for third-party components of this work must be honored.

For all other uses, contact the owner/author(s).

HotSoS, April 1-3, 2019, Nashville, TN, USA

(C) 2019 Copyright held by the owner/author(s)

ACM ISBN 978-1-4503-7147-6/19/04.

https://doi.org/10.1145/3314058.3317726

\section{INTRODUCTION}

Increasing connectivity and variability of DERs have made modern power-grids not only capable of being managed highly efficiently but also vulnerable to disruptions and cyber-physical attacks. In addition, Transactive Energy (TE) approaches [3] are now being integrated into grid operations to manage them efficiently. In general, modern grids can have not only a large number of connected physical grid components but also several computational and communication components that make the grid operations possible. In order to evaluation such grid operations and study their resilience against different kinds of cyber-physical attacks, a comprehensive evaluation platform is needed.

In this research, we present a web-based design and simulation platform (called a design studio) for evaluation of power-grid distribution systems and transactive energy approaches [1]. The platform provides a novel Domain-Specific Modeling Language (DSML) using the Web-based Generic Modeling Environment (WebGME [4]) for the graphical modeling of power-grid, cyber-physical attacks, and TE scenarios. A cloud-hosted simulation backend using the GridLAB-D power-grid distribution system simulation tool [2] is also provided. Using WebGME, the platform provides a DSML that can be used for modeling power-grid models based on the grid modeling semantics of the GridLAB-D power-grid simulation. The platform allows power-grid researchers to:

- Import any GridLAB-D network model textual code (.glm file) into the modeling environment to visualize the network graphically that shows a clear picture of the grid,

- Edit the imported model in the graphical model editor and generate the corresponding GridLAB-D model file,

- Create a new model of the power-grid from scratch in an easy-to-use environment and generate syntactically correct model file (.glm file) through code-generation,

- Simulate the model directly from the web-based platform,

- Retreive simulation outputs as a packaged zip file,

- Experiment with market models and attack scenarios,

- Select and configure models from model library,

- Version control (as in git) the models and experiments,

- Collaborate using multi-user real-time model editing, and

- Extend the platform by creating their own library of grid and attack models with pre-defined values according to their particular experiment needs. 


\section{ARCHITECTURE}

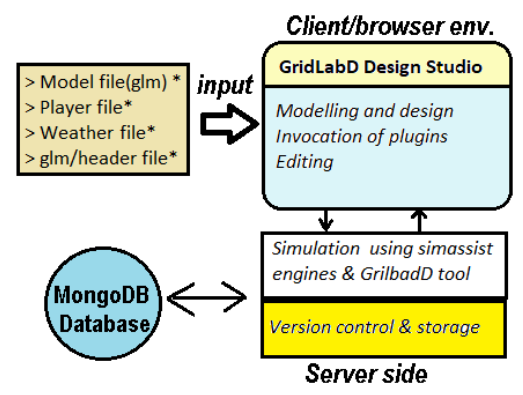

Figure 1: Platform Architecture

Our power-grid and TE evaluation platform is based on WebGME [4]), which is a web- and cloud- based collaborative, scalable meta-modeling tool. WebGME supports creating graphical domainspecific modeling languages, multi-user model editing, and integrating model-interpretation tools (aka. plugins). We applied these to create a platform for modeling and analyzing power-grids.

As shown in Fig. 1, the tool has three major sections: (1) Clien$\boldsymbol{t} /$ Web Browser (the officially supported browser is Chrome), (2) Simulation Backend Server, and (3) MongoDB object-oriented database. The web-browser is the interface where user interacts with tool. User can invoke the platform's plugins to access various features supported by the platform. MongoDB database is configured to store model updates by all users in a git like model repository as well as simulation outputs. After finalizing grid model, the user can invoke the plugin 'SimulateWithGridlabD'. The plugin:

- Loads grid model and converts into GridLAB-D model file,

- Generates different parameter files and Python scripts,

- Sends everything to the backend simulation server,

- Invokes and manages the simulation engines (simassist and GridLAB-D). Our Simassist engine, written in Python, configures and controls simulation, and

- Displays simulation progress and provides outputs in a packaged zip file as soon as the simulation is finished.

\section{DEMONSTRATION}

Our platform [1] has been demonstrated with above-mentioned functionality with various distribution grid models and experiment scenarios (e.g., attacks on market, attacks on bids, and weathersensitive power generation using solar-panels). Fig. 2 illustrates a small part of a grid model. The full grid model consists of 53 houses as well as nodes connected through overhead and underground lines. Each node has a triplex-meter connected to it via a transformer. The grid also uses intermediate step-up and step-down transformers. Each house has a zip-load, a water-heater, and a transactive controller. The controller interacts with the central market and controls the house parameters (e.g., temperature setpoint). We ran the grid simulation for a day and used weather data of Nashville, TN, USA and a compromised market where all controllers participated.

We designed two scenarios to demonstrate attacks that either inject false data in the system or manipulate control commands. In scenario-1, the attacker creates a demand spike via attacks on markets. In scenario-2, the attacker manipulates market's equilibrium to achieve higher market clearing prices. For brevity, only scenario- 1 is described below.

In scenario-1, the attacker suddenly exceedingly increases seller bid price for 1 hour. Consequently, none of the controllers buy power and the room temperatures increase with air-conditioners in OFF state. The attacker than abruptly lowers the power price for 30 minutes causing a sudden influx of power demand. This results in a demand spike about 2-3 times the normal demand. This can be highly catastrophic and even in modern grids can cause sudden frequency drops, which in-turn can set-off substation circuit-breakers and potentially collapse significant part of the grid. Fig. 3 clearly shows the resulting peak demand in this experiment.

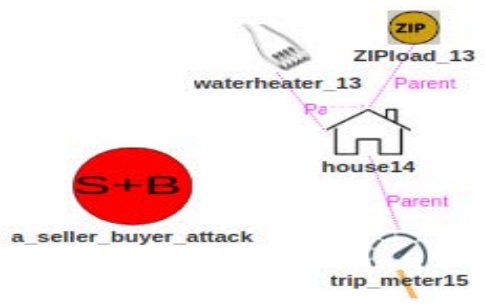

Figure 2: Grid Modeling: Illustrative Example

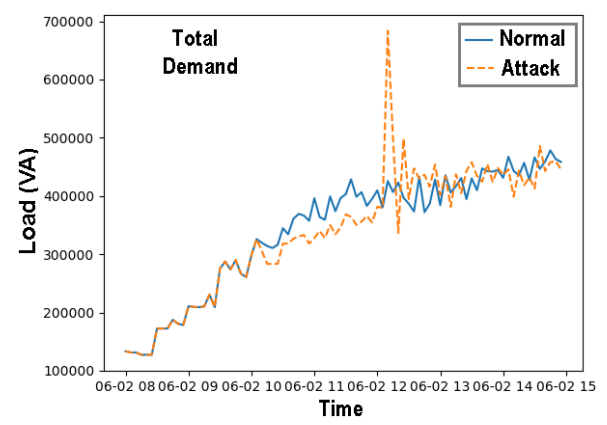

Figure 3: Attack Scenario-1: Demand Peak Creation

The range of modeling and experimentation features provided and the capability to extend the model library makes this platform an effective tool for evaluating power-grids, market models, transactive energy approaches, and cyber-physical attack scenarios.

\section{ACKNOWLEDGMENTS}

This work is supported by in part by the following grants: NSF FORCES \#CNS-1238959, NSF PIRE and CPS \#1521617, and NIST \#70NANB18H269 \& \#70NANB17H266.

\section{REFERENCES}

[1] 2019. Power-grid analysis framework. (2019). https://cps-vo.org/group/gridlabd

[2] David P Chassin, K Schneider, and C Gerkensmeyer. 2008. GridLAB-D: An opensource power systems modeling and simulation environment. In Transmission and distribution conference and exposition, 2008. $t \& d$. IEEE/PES. IEEE, 1-5.

[3] GridWise Architecture Council. 2015. GridWise Transactive Energy Framework: Version 1.0. Pacific Northwest National Laboratory, PNNL-22946 Ver 1.0 (2015).

[4] Miklós Maróti, Róbert Kereskényi, Tamás Kecskés, Péter Völgyesi, and Akos Lédeczi. 2014. Online collaborative environment for designing complex computational systems. Procedia Computer Science 29 (2014), 2432-2441. 